\title{
Effective Empirical Acceleration Modeling and its Application to Enhanced-Accuracy Orbit Prediction
}

\author{
By Wen-bin WANG ${ }^{1,2)}$ and Yang GAO ${ }^{1,2)}$ \\ ${ }^{1)}$ Technology and Engineering Center for Space Utilization, Chinese Academy of Sciences, Beijing, China \\ ${ }^{2)}$ Key Laboratory of Space Utilization, Chinese Academy of Sciences, Beijing, China
}

(Received July 31 st, 2015)

\begin{abstract}
Effective computing methods are proposed for calculating the sensitivity matrices related to empirical acceleration parameters that are used to compensate for dynamic modeling deficiencies. Meanwhile, design and normal equation matrices are expressed in the form of partitioned sub-matrices. These methods of computing the sensitivity matrices and partitioning the normal equation matrices are extremely timesaving and require less storage, compared to conventional methods. The GRACE real flight GPS observation data has been used to evaluate the computing performance of setting up normal equation. The efficiency increased to 2 times when using code observations only, and increased to 3.5 times when using code and carrier phase observations together. Furthermore, we found that the empirical accelerations showed quasi-periodic characteristics with respect to time variable, which allowed Fourier series to be used to interpolate empirical accelerations. The fitted tangential empirical acceleration curves, which are added into deterministic force models, form enhanced-accuracy dynamic models that are applied to orbit prediction. For 3-day orbit pass, the prediction accuracy was better than 60 meters and an improvement of 2.3 times on average was achieved, compared to conventional dynamic models without considering empirical acceleration models. The proposed prediction schemes are beneficial to the establishment of advanced, possibly onboard, satellite navigation systems.
\end{abstract}

Key Words: Reduced Dynamic, Empirical Accelerations, Partitioned Sub-matrices, Fourier Series, Orbit Prediction

\section{Nomenclature}

$\begin{array}{lll}t & : & \text { time } \\ \boldsymbol{r} & : & \text { position } \\ \boldsymbol{v} & : & \text { velocity } \\ \boldsymbol{y}(t) & : & \text { position and velocity } \\ \boldsymbol{y}_{0} & : & \boldsymbol{y}\left(t_{0}\right) \\ C_{D} & : & \text { atmospheric drag coefficient } \\ C_{R} & : & \text { solar radiation coefficient } \\ \boldsymbol{e}_{R} & : & \text { unit vector of radial direction } \\ \boldsymbol{e}_{T} & : & \text { unit vector of along-track direction } \\ \boldsymbol{e}_{N} & : \text { unit vector of cross-track direction } \\ \tau & : \text { length of subinterval } \\ n & : \text { number of empirical accelerations } \\ n_{\text {obs }} & : \text { number of GPS observations } \\ \text { LSQ } & : \text { Least Squares }\end{array}$

\section{Introduction}

The deterministic force models are not accurate enough for LEO satellites due to the complex properties and dynamic of the earth's atmosphere. Meanwhile, the accuracy of GPS observations (code and carrier phase observations) has reached to the level of millimeters. Deterministic force models of real-world LEO satellites are hardly known to a level of accuracy that matches that of GPS observations. ${ }^{1,2)}$ Thus, the accuracy of orbit determination mainly depends on the GPS observations. The concept of reduced dynamic orbit determination has been introduced in previous decades. ${ }^{3,4)}$ The deterministic force models are compensated by the introduction of stochastic parameters, which take the form of empirical accelerations or instantaneous velocity pulses, form reduced dynamic models. The stochastic parameters are adjusted along with other parameters in the course of orbit determination process. Thus the reduced dynamic models can match the accuracy of GPS observations without losing the robustness provided by deterministic dynamic models.

Empirical accelerations are considered to be piecewise constant in pre-defined subintervals. The along-track accelerations mainly reflect the mismodeling of atmospheric drag. Nowadays, empirical accelerations are widely used in the reduced dynamic batch least squares (LSQ) processes and orbit accuracy has reached centimeters level. ${ }^{1,5-7)}$ From the view point of efficiency, one has to solve one variational equation for each parameter and for each observation epoch. So the disadvantage is that the scale of numerical integration and storage related to sensitivity matrices is very large. In addition, it takes a long time to set up the normal equations if more parameters and GPS observations are involved when using conventional methods.

Empirical accelerations reflect overall errors of the deterministic force models, and can have an enhancing effect on orbit prediction if the main features of unmodeled errors can be modeled properly. Jaggi ${ }^{8)}$ applied empirical accelerations to GOCE orbit prediction during the period of 
drag-free flight. He compared three orbit prediction strategies: the first was a "straight-forward" strategy, it used deterministic models to propagate orbit only; the second was a "once-per-rev" strategy, its orbit extrapolation was based on deterministic models and once-per-revolution parameters, ${ }^{9)}$ which were estimated as a part of the orbit determination; and the last was a "empirical" strategy, that was similar to the second strategy but used empirical accelerations parameters, which were in form modeled as a function of the argument of latitude. The "empirical" strategy provided the best results with a mean along-track deviation of $267 \mathrm{~m}$ after prediction of 12 hours. The "straight-forward" and "once-per-rev" strategy gave inferior results with mean along-track deviations of 465 $\mathrm{m}$ and $508 \mathrm{~m}$, respectively.

This paper has two aims. One is to improve computational efficiency in the reduced dynamic batch LSQ process. This paper proposes an effective modeling method for empirical accelerations. Thus, the normal equation can be expressed in the form of partitioned sub-matrices. The other one is to improve accuracy of orbit prediction. The empirical acceleration shows quasi-periodic characteristics with respect to time variable, which can be in form modeled by a Fourier series. Enhanced-accuracy dynamic models are proposed and tested with GPS observations collected onboard the GRACE satellite to assess orbit prediction. The two aims both relate to the empirical accelerations.

\section{Reduced Dynamic Modeling}

\subsection{Empirical accelerations}

The whole observation arc $\left[t_{0}, t_{n}\right]$ is divided into $n$ subintervals averagely. Empirical accelerations $\boldsymbol{a}_{i}=\left(a_{i R}, a_{i T}, a_{i N}\right)^{\mathrm{T}} \quad$ keep constant in subintervals $\left[t_{i}, t_{i+1}\right)$ $\left(t_{i}=t_{0}+i \tau, i=0,1, \cdots, n-1\right)$ with directions of $\boldsymbol{e}_{R}, \boldsymbol{e}_{T}$, and $\boldsymbol{e}_{N}$, respectively. When taking empirical accelerations parameters into the orbit, the equation of motion reads

$$
\begin{aligned}
& \ddot{\boldsymbol{r}}(t)=\boldsymbol{a}(t, \boldsymbol{r}, \boldsymbol{v})+ \\
& \xi_{i}(t)\left(a_{i R} \boldsymbol{e}_{R}(t)+a_{i T} \boldsymbol{e}_{T}(t)+a_{i N} \boldsymbol{e}_{N}(t)\right)
\end{aligned}
$$

where $\boldsymbol{a}$ is the deterministic forces, and $\xi_{i}(t)$ is defined as

$$
\xi_{i}(t)=\left\{\begin{array}{lc}
0 & t<t_{i} \\
1 & t_{i} \leq t<t_{i+1} \\
0 & t_{i+1} \leq t
\end{array}\right.
$$

The partial derivatives of $\boldsymbol{y}(t)$ with respect to $\boldsymbol{a}_{i}$ are denoted as the sensitivity matrix, $\boldsymbol{S}_{a_{i}}(t)$. The variational equation $\boldsymbol{S}_{a_{i}}(t)$ reads

$$
\frac{\mathrm{d}}{\mathrm{d} t} \boldsymbol{S}_{a_{i}}(t)=\boldsymbol{M}(t, \boldsymbol{r}, \boldsymbol{v}) \boldsymbol{S}_{a_{i}}(t)+\left[\begin{array}{ccc}
\mathbf{0} & \\
\boldsymbol{e}_{R}(t) & \boldsymbol{e}_{T}(t) & \boldsymbol{e}_{N}(t)
\end{array}\right]
$$

where the matrix $\boldsymbol{M}$ is the Jacobian matrix of $\boldsymbol{a}$ with respect to the $\boldsymbol{r}$ and $\boldsymbol{v}$ of the satellite. The solution of Eq. (3) can be expressed as ${ }^{7)}$

$$
\boldsymbol{S}_{a_{i}}(t)=\left\{\begin{array}{lr}
\mathbf{0} & t<t_{i} \\
\int_{t_{i}}^{t} \frac{\mathrm{d}}{\mathrm{d} t} \boldsymbol{S}_{a_{i}}(t) \mathrm{d} t & t_{i} \leq t<t_{i+1} \\
\boldsymbol{\Phi}\left(t, t_{0}\right) \boldsymbol{P}_{i+1} & t_{i+1} \leq t
\end{array}\right.
$$

We distinguish the solutions to three regimes,

1) where $\boldsymbol{S}_{a_{i}}(t)$ is zero for previous intervals $\left(t<t_{i}\right)$, i.e. $\boldsymbol{a}_{i}$ has no effect on previous satellite states,

2) where $\boldsymbol{S}_{a_{i}}(t)$ is computed by numerical integration according to differential equation of Eq. (3) with initial value of zero when $t_{i} \leq t<t_{i+1}$,

3) where $\boldsymbol{S}_{a_{i}}(t)$ is expressed as state transition matrix $\boldsymbol{\Phi}\left(t, t_{0}\right)$ times constant matrix $\boldsymbol{P}_{i+1}$ for after intervals $\left(t_{i+1} \leq t\right) . \quad \boldsymbol{P}_{i+1} \quad$ can be written as

$$
\boldsymbol{P}_{i+1}=\boldsymbol{\Phi}^{-1}\left(t_{i+1}, t_{0}\right) \boldsymbol{S}_{a_{i}}\left(t_{i+1}\right)
$$

Since $\boldsymbol{S}_{a_{i}}(t)$ is continuous over the whole arc, $\boldsymbol{S}_{a_{i}}\left(t_{i+1}\right)$ equals the integral of Eq. (3) from $t_{i}$ to $t_{i+1}$. We notice that Eq. (3) is homogeneous for $\boldsymbol{a}_{i}$ when $t \geq t_{i+1}$, so it is not necessary to compute all sensitivity matrices by numerical integration. The above described Eq. (4) for determining the sensitivity matrices is extremely time-saving and requires less storage when compared with conventional method without using the homogeneous characteristic of variational equations.

Fig. 1 illustrates the activity areas of sensitivity matrices related to empirical acceleration parameters. Blank areas are zero. Gray areas indicate that sensitivity matrices can be expressed as state transition matrices times constant matrices, i.e. they do not require the time-consuming integration processes any more. Moreover, these areas account for a significant portion of the matrix. " $\times$ " areas indicate that the sensitivity matrices are computed by numerical integration in terms of Eq. (3). Typically, in the conventional method all the gray and $" \times "$ areas would be computed by numerical integration. We observe a roughly linear growth of the number of active accelerations with time, implying a quadratic growth in the storage requirement and sensitivity matrices computation for the conventional modeling method. The computing method presented here makes the storage requirement and the integration of sensitivity matrices increase with time linearly.

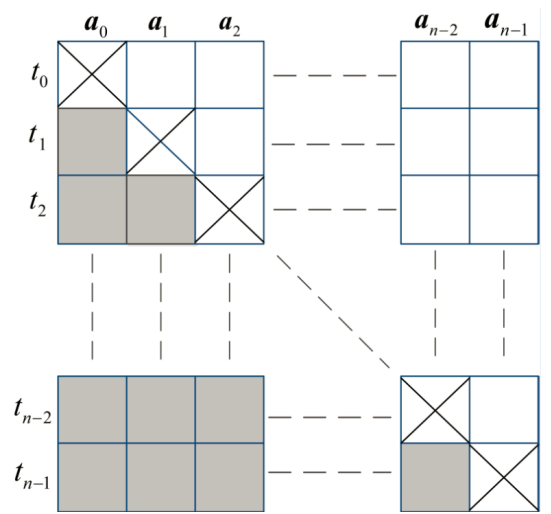

Fig. 1. The activity areas of sensitivity matrices related to empirical accelerations parameters. 


\subsection{Partitioned sub-matrix modeling}

The setting up of normal equation for empirical accelerations can be done efficiently using the above relationships between state transition matrices and sensitivity matrices. Denoting $\boldsymbol{z}_{i}, \boldsymbol{h}_{i}$ and $\boldsymbol{\varepsilon}_{i}$ as observations, observation model values, and residual values of the observations in subinterval $\left[t_{i}, t_{i+1}\right)$, respectively, $i=0,1, \cdots, n-1$. While linearizing the observation models $\boldsymbol{h}_{i}$ with respect to $\boldsymbol{y}_{0}$ and empirical acceleration parameters $\boldsymbol{a}_{i}$, we get:

$$
\boldsymbol{z}_{i}=\boldsymbol{h}_{i}+\frac{\partial \boldsymbol{h}_{i}}{\partial \boldsymbol{y}_{0}} \Delta \boldsymbol{y}_{0}+\sum_{m=0}^{i} \frac{\partial \boldsymbol{h}_{i}}{\partial \boldsymbol{a}_{m}} \Delta \boldsymbol{a}_{m}-\boldsymbol{\varepsilon}_{i}
$$

Empirical accelerations are divided into two parts and the solutions of sensitivity matrices are introduced using the Eq. (4), we get

$$
\boldsymbol{H}_{i} \Delta \boldsymbol{y}_{0}+\boldsymbol{H}_{i} \sum_{m=0}^{i-1} \boldsymbol{P}_{m+1} \Delta \boldsymbol{a}_{m}+\overline{\boldsymbol{P}}_{i} \Delta \boldsymbol{a}_{i}-\boldsymbol{b}_{i}=\boldsymbol{\varepsilon}_{i}
$$

where $\boldsymbol{b}_{i}=\boldsymbol{z}_{i}-\boldsymbol{h}_{i}$ denotes the difference between the observed values and the computed values. Here $\boldsymbol{H}_{i}=\partial \boldsymbol{h}_{i} / \partial \boldsymbol{y}_{0}$ is the design matrix, $\boldsymbol{P}_{m+1}$ is evaluated according to Eq. (5), and

$$
\overline{\boldsymbol{P}}_{i}=\frac{\partial \boldsymbol{h}_{i}}{\partial \boldsymbol{a}_{i}}=\frac{\partial \boldsymbol{h}_{i}}{\partial \boldsymbol{y}_{i}} \boldsymbol{S}_{a_{i}}(t) \quad t_{i} \leq t<t_{i+1}
$$

where $S_{a_{i}}(t)$ is obtained by integration according to variational equation Eq. (3).

The correction equation $\boldsymbol{H} \Delta \boldsymbol{x}=\boldsymbol{b}$ for the whole observations, where all the observations are defined in subintervals $\left[t_{i}, t_{i+1}\right), i=0,1, \cdots, n-1$, can be written as partitioned sub-matrices

$$
\left[\begin{array}{cccccc}
\boldsymbol{H}_{0} & \overline{\boldsymbol{P}}_{0} & \mathbf{0} & \cdots & \mathbf{0} & \mathbf{0} \\
\boldsymbol{H}_{1} & \boldsymbol{H}_{1} \boldsymbol{P}_{1} & \overline{\boldsymbol{P}}_{1} & \cdots & \mathbf{0} & \mathbf{0} \\
\boldsymbol{H}_{2} & \boldsymbol{H}_{2} \boldsymbol{P}_{1} & \boldsymbol{H}_{2} \boldsymbol{P}_{2} & \cdots & \mathbf{0} & \mathbf{0} \\
\vdots & \vdots & \vdots & \ddots & \vdots & \vdots \\
\boldsymbol{H}_{n-2} & \boldsymbol{H}_{n-2} \boldsymbol{P}_{1} & \boldsymbol{H}_{n-2} \boldsymbol{P}_{2} & \cdots & \overline{\boldsymbol{P}}_{n-2} & \mathbf{0} \\
\boldsymbol{H}_{n-1} & \boldsymbol{H}_{n-1} \boldsymbol{P}_{1} & \boldsymbol{H}_{n-1} \boldsymbol{P}_{2} & \cdots & \boldsymbol{H}_{n-1} \boldsymbol{P}_{n-1} & \overline{\boldsymbol{P}}_{n-1}
\end{array}\right] \cdot \Delta \boldsymbol{x}=\left[\begin{array}{c}
\boldsymbol{b}_{0} \\
\boldsymbol{b}_{1} \\
\boldsymbol{b}_{2} \\
\vdots \\
\boldsymbol{b}_{n-2} \\
\boldsymbol{b}_{n-1}
\end{array}\right]
$$

where

$$
\Delta \boldsymbol{x}^{\mathrm{T}}=\left(\Delta \boldsymbol{y}_{0}^{\mathrm{T}}, \Delta \boldsymbol{a}_{0}^{\mathrm{T}}, \cdots, \Delta \boldsymbol{a}_{n-1}^{\mathrm{T}}\right)
$$

Accordingly, the full normal equation $\boldsymbol{H}^{\mathrm{T}} \boldsymbol{W} \boldsymbol{H} \Delta \boldsymbol{x}=\boldsymbol{H}^{\mathrm{T}} \boldsymbol{W} \boldsymbol{b}$ is written as two parts

$$
\begin{aligned}
& \boldsymbol{H}^{\mathrm{T}} \boldsymbol{W} \boldsymbol{H}=\boldsymbol{M}_{1}+\boldsymbol{M}_{2} \\
& \boldsymbol{H}^{\mathrm{T}} \boldsymbol{W} \boldsymbol{b}=\boldsymbol{l}_{1}+\boldsymbol{l}_{2}
\end{aligned}
$$

where the weighting matrix $\boldsymbol{W}$ is block-diagonal, given by the inverse of observations error deviation. The observations may compose different measurement types. The accuracy of each measurement type may easily be account for by adding weighting factor.

Here $\boldsymbol{M}_{1}$ and $\boldsymbol{l}_{1}$ are written as

$$
\boldsymbol{M}_{1}=\left[\begin{array}{ccccc}
\boldsymbol{N}_{00} & \boldsymbol{N}_{10}^{\mathrm{T}} & \cdots & \boldsymbol{N}_{n-1,0}^{\mathrm{T}} & \mathbf{0} \\
\boldsymbol{N}_{10} & \boldsymbol{N}_{11} & \cdots & \boldsymbol{N}_{n-1,1}^{\mathrm{T}} & \mathbf{0} \\
\vdots & \vdots & \ddots & \vdots & \vdots \\
\boldsymbol{N}_{n-1,0} & \boldsymbol{N}_{n-1,1} & \cdots & \boldsymbol{N}_{n-1, n-1} & \mathbf{0} \\
\mathbf{0} & \mathbf{0} & \cdots & \mathbf{0} & \mathbf{0}
\end{array}\right]
$$

and

$$
\boldsymbol{l}_{1}^{\mathrm{T}}=\left(\boldsymbol{L}_{0}^{\mathrm{T}}, \boldsymbol{L}_{1}^{\mathrm{T}}, \cdots, \boldsymbol{L}_{n-1}^{\mathrm{T}}, \mathbf{0}^{\mathrm{T}}\right)
$$

respectively, where $\quad \boldsymbol{N}_{i j}=\boldsymbol{P}_{i}^{\mathrm{T}} \sum_{k=i}^{n-1} \boldsymbol{H}_{k}^{\mathrm{T}} \boldsymbol{W}_{k} \boldsymbol{H}_{k} \boldsymbol{P}_{j}$ $\boldsymbol{L}_{i}=\boldsymbol{P}_{i}^{\mathrm{T}} \sum_{k=i}^{n-1} \boldsymbol{H}_{k}^{\mathrm{T}} \boldsymbol{W}_{k} \boldsymbol{b}_{k} \quad, \quad i, j=0,1, \cdots, n-1$. We $\quad$ set $\quad \boldsymbol{P}_{0} \quad$ as identity matrix and $\boldsymbol{W}_{i}$ as weighting matrix pertaining to $\left[t_{i}, t_{i+1}\right)$. The terms that related to $\overline{\boldsymbol{P}}_{i}$ represents as

$$
\boldsymbol{M}_{2}=\left[\begin{array}{ccccc}
\mathbf{0} & \boldsymbol{Q}_{00}^{\mathrm{T}} & \boldsymbol{Q}_{10}^{\mathrm{T}} & \cdots & \boldsymbol{Q}_{n-1,0}^{\mathrm{T}} \\
\boldsymbol{Q}_{00} & \overline{\boldsymbol{P}}_{0}^{\mathrm{T}} \boldsymbol{W}_{0} \overline{\boldsymbol{P}}_{0} & \boldsymbol{Q}_{11}^{\mathrm{T}} & \cdots & \boldsymbol{Q}_{n-1,1}^{\mathrm{T}} \\
\boldsymbol{Q}_{10} & \boldsymbol{Q}_{11} & \overline{\boldsymbol{P}}_{1}^{\mathrm{T}} \boldsymbol{W}_{1} \overline{\boldsymbol{P}}_{1} & \ldots & \boldsymbol{Q}_{n-1,2}^{\mathrm{T}} \\
\vdots & \vdots & \vdots & \ddots & \vdots \\
\boldsymbol{Q}_{n-1,0} & \boldsymbol{Q}_{n-1,1} & \boldsymbol{Q}_{n-1,2} & \cdots & \overline{\boldsymbol{P}}_{n-1}^{\mathrm{T}} \boldsymbol{W}_{n-1} \overline{\boldsymbol{P}}_{n-1}
\end{array}\right]
$$

and

$$
\boldsymbol{l}_{2}=\left[\begin{array}{c}
\mathbf{0} \\
\overline{\boldsymbol{P}}_{0}^{\mathrm{T}} \boldsymbol{W}_{0} \boldsymbol{b}_{0} \\
\overline{\boldsymbol{P}}_{1}^{\mathrm{T}} \boldsymbol{W}_{1} \boldsymbol{b}_{1} \\
\vdots \\
\overline{\boldsymbol{P}}_{n-2}^{\mathrm{T}} \boldsymbol{W}_{n-2} \boldsymbol{b}_{n-2} \\
\overline{\boldsymbol{P}}_{n-1}^{\mathrm{T}} \boldsymbol{W}_{n-1} \boldsymbol{b}_{n-1}
\end{array}\right]
$$

respectively, where $\boldsymbol{Q}_{i j}=\overline{\boldsymbol{P}}_{i} \boldsymbol{W}_{i} \boldsymbol{H}_{i} \boldsymbol{P}_{j}, i, j=0,1, \cdots, n-1$.

The full normal equation system may thus be set up easily and efficiently:

1) There are no large matrices involved when setting up these matrices. The computational effect, when compared with conventional method without applying partitioned sub-matrices, is enormous. See Section 3 for an illustration with real flight data.

2) The basic building blocks of $\boldsymbol{M}_{1}$ are $\sum_{i=m}^{n-1} \boldsymbol{H}_{i}^{\mathrm{T}} \boldsymbol{W}_{i} \boldsymbol{H}_{i}$ and $\sum_{i=m}^{n-1} \boldsymbol{H}_{i}^{\mathrm{T}} \boldsymbol{W}_{i} \boldsymbol{b}_{i}, m=n-1, n-2, \cdots, 0$. As indicated by this reversed sequence, these sums are best built up starting with $m=n-1$ and ending up with $m=0$.

3) The sub-matrix $\boldsymbol{N}_{m n}$ is obtained by multiplying the sum $\sum_{i=m}^{n-1} \boldsymbol{H}_{i}^{\mathrm{T}} \boldsymbol{W}_{i} \boldsymbol{H}_{i} \quad$ with matrix $\boldsymbol{P}_{m}^{\mathrm{T}}$ from the left and with matrix $\boldsymbol{P}_{n}$ from the right for $m=n-1, n-2, \cdots, 0$. Sub-vector $\boldsymbol{L}_{m}$ is obtained by multiplying the sum $\sum_{i=m}^{n-1} \boldsymbol{H}_{i}^{\mathrm{T}} \boldsymbol{W}_{i} \boldsymbol{b}_{i}$ with $\quad \boldsymbol{P}_{m}^{\mathrm{T}}$ from the left.

The normal equation for the conventional method is set up 
by the multiplication of matrices with dimension of $(6+3 n) \times n_{o b s}$, its efficiency will decrease greatly when many GPS observations and acceleration parameters are involved. The saving of processing time is significant when setting up normal equation using the partitioned sub-matrices method, because it makes use of the relationship between the state transition matrices and sensitivity matrices to cut down large repeated and invalid computations. The comparisons of efficiency between conventional method and this partitioned sub-matrices method will be discussed in Section 3.

\subsection{Reduced dynamic modeling for GPS-based observations}

When estimating an orbit using the GPS ionosphere free code and carrier phase observations of satellite receiver, one has to cope with clock offsets and ambiguity parameters in addition to initial states and acceleration parameters. For LEOs, $C_{D}$ and $C_{R}$ have to be considered in the dynamic models.

The estimation parameters are grouped into the $n_{T}$-dimensional vector of GPS receiver clock offsets

$$
\boldsymbol{T}=\left(c \delta t_{0}, \ldots, c \delta t_{n_{T}-1}\right)^{\mathrm{T}}
$$

the $(8+3 n)$-dimensional vector concerning the dynamic estimation parameters

$$
\boldsymbol{Y}=\left(\boldsymbol{y}_{0}{ }^{\mathrm{T}}, C_{D}, C_{R}, \boldsymbol{a}_{0}{ }^{\mathrm{T}}, \ldots, \boldsymbol{a}_{n-1}{ }^{\mathrm{T}}\right)^{\mathrm{T}}
$$

and the $n_{B}$-dimensional vector of ionosphere free carrier phase ambiguity parameters

$$
\boldsymbol{B}=\left(B_{0}, \ldots, B_{n_{B}-1}\right)^{\mathrm{T}}
$$

Accordingly, a linearization around initial values $\left(\boldsymbol{T}_{0}, \boldsymbol{Y}_{0}, \boldsymbol{B}_{0}\right)$ for the estimation parameters takes place

$$
\begin{aligned}
& \boldsymbol{T}=\boldsymbol{T}_{0}+\Delta \boldsymbol{T} \\
& \boldsymbol{Y}=\boldsymbol{Y}_{0}+\Delta \boldsymbol{Y} \\
& \boldsymbol{B}=\boldsymbol{B}_{0}+\Delta \boldsymbol{B}
\end{aligned}
$$

and the updates are computed in reduced dynamic batch LSQ process, given by

$$
\begin{aligned}
& {\left[\frac{\partial \boldsymbol{h}}{\partial\left(\boldsymbol{T}_{0}, \boldsymbol{Y}_{0}, \boldsymbol{B}_{0}\right)}\right]^{\mathrm{T}} \boldsymbol{W}\left[\frac{\partial \boldsymbol{h}}{\partial\left(\boldsymbol{T}_{0}, \boldsymbol{Y}_{0}, \boldsymbol{B}_{0}\right)}\right]\left[\begin{array}{c}
\Delta \boldsymbol{T} \\
\Delta \boldsymbol{Y} \\
\Delta \boldsymbol{B}
\end{array}\right]} \\
& =\left[\frac{\partial \boldsymbol{h}}{\partial\left(\boldsymbol{T}_{0}, \boldsymbol{Y}_{0}, \boldsymbol{B}_{0}\right)}\right]^{\mathrm{T}} \boldsymbol{W}\left(\boldsymbol{z}-\boldsymbol{h}\left(\boldsymbol{T}_{0}, \boldsymbol{Y}_{0}, \boldsymbol{B}_{0}\right)\right)
\end{aligned}
$$

The overall design matrix

$$
\left[\frac{\partial \boldsymbol{h}}{\partial\left(\boldsymbol{T}_{0}, \boldsymbol{Y}_{0}, \boldsymbol{B}_{0}\right)}\right]=\left(\boldsymbol{H}_{T} \boldsymbol{H}_{\boldsymbol{Y}} \boldsymbol{H}_{\boldsymbol{B}}\right)
$$

is constructed by three partitioned ones. According to the literature 6), Eq. (20) can furthermore be reduced to

$$
\left(\begin{array}{lll}
\boldsymbol{N}_{T T} & \boldsymbol{N}_{T Y} & \boldsymbol{N}_{T B} \\
\boldsymbol{N}_{Y T} & \boldsymbol{N}_{Y Y} & \boldsymbol{N}_{Y B} \\
\boldsymbol{N}_{B T} & \boldsymbol{N}_{B Y} & \boldsymbol{N}_{B B}
\end{array}\right)\left(\begin{array}{c}
\Delta \boldsymbol{T} \\
\Delta \boldsymbol{Y} \\
\Delta \boldsymbol{B}
\end{array}\right)=\left(\begin{array}{c}
\Delta \boldsymbol{n}_{T} \\
\Delta \boldsymbol{n}_{Y} \\
\Delta \boldsymbol{n}_{B}
\end{array}\right)
$$

where $\quad \boldsymbol{N}_{\boldsymbol{P Q}}=\boldsymbol{H}_{\boldsymbol{P}}^{\mathrm{T}} \boldsymbol{W} \boldsymbol{H}_{\boldsymbol{Q}} \quad$ and $\quad \Delta \boldsymbol{n}_{\boldsymbol{P}}=\boldsymbol{H}_{\boldsymbol{P}}^{\mathrm{T}} \boldsymbol{W}\left(\boldsymbol{z}-\boldsymbol{h}\left(\boldsymbol{T}_{0}, \boldsymbol{Y}_{0}, \boldsymbol{B}_{0}\right)\right.$,
$\boldsymbol{P}$ and $\boldsymbol{Q}$ denote one of $\boldsymbol{T}, \boldsymbol{Y}$ and $\boldsymbol{B}$.

The most time-consuming part in Eq. (22) is to set up $\boldsymbol{N}_{Y Y}$. In order to make use of the partitioned sub-matrices modeling method as described in section 2.2, all sub-matrices $\boldsymbol{H}_{i}$ in Eq. (9) have to be expanded by 2 columns for the partial derivatives with respect to $C_{D}$ and $C_{R}$, which are written as

$$
\boldsymbol{H}_{i}^{+}=\left[\boldsymbol{H}_{i} \frac{\partial \boldsymbol{h}_{i}}{\partial C_{D}} \frac{\partial \boldsymbol{h}_{i}}{\partial C_{R}}\right]
$$

Accordingly, the sub-matrices $\boldsymbol{P}_{i}$ in Eq. (9) have to be expanded by 2 lines, denoted as

$$
\boldsymbol{P}_{i}^{+}=\left[\begin{array}{c}
\boldsymbol{P}_{i} \\
\mathbf{0}^{\mathrm{T}} \\
\mathbf{0}^{\mathrm{T}}
\end{array}\right]
$$

sub-matrices $\overline{\boldsymbol{P}}_{i}$ are not changed. The sub-matrix related to $\boldsymbol{Y}$ is completed by exchanging the $\boldsymbol{H}_{i}$ and $\boldsymbol{P}_{i}$ by matrices $\boldsymbol{H}_{i}^{+}$and $\boldsymbol{P}_{i}^{+}$. The structure of the design and normal equation matrix are not changed when compared with the design matrix in Eq. (9) and normal equation matrix in Eq. (11).

\section{Analysis of Computational Efficiency}

One entire day of GRACE-A GPS data (Jan. 3, 2006) with sampling interval of 30 seconds was processed, where we focused on the efficiency of setting up the normal equation

\begin{tabular}{|c|c|}
\hline Dynamic models & Description \\
\hline \multirow[t]{2}{*}{ Static gravity field } & GGM01C $(120 \times 120)^{12)}$ \\
\hline & Solid Earth tide (IERS 2003) \\
\hline \multirow[t]{2}{*}{ Tidal perturbations } & Ocean tide (UT/CSR3.0) \\
\hline & Polar tide (IERS 2003) \\
\hline \multirow[t]{2}{*}{ 3th body gravity } & $\begin{array}{l}\text { Analytical series expansions of luni-solar } \\
\text { coordinates }{ }^{13)}\end{array}$ \\
\hline & NRLMSISE $2000^{14)}$ \\
\hline \multirow[t]{2}{*}{ Atmospheric drag } & NOAA solar flux (daily) \\
\hline & Geomagnetic activity ( 3 hourly) \\
\hline Solar radiation pressure & Conical earth shadow ${ }^{13)}$ \\
\hline Albedo & $\begin{array}{l}\text { Shortwave optical radiation and longwave } \\
\text { infrared radiation }{ }^{15)}\end{array}$ \\
\hline
\end{tabular}
system.

The GRACE-A GPS observations and reference ephemerides are obtained from JPLs Physical Oceanography Distributed Active Archive Center (PODAAC). ${ }^{10)}$ The CODE high rate clocks and accompanying orbits ${ }^{11)}$ are used for precise orbit determination. We set the length of subinterval $\tau$ to be 600 seconds, so the number of empirical accelerations was 432 . The deterministic force models were showed in Table 1, and RKF78 integrator with fixed step size of $30 \mathrm{~s}$ was chosen for numerical integration.

Table 1. Deterministic dynamic models.

We selected two kinds of ionosphere free combinations for test. One is code observations, which only contain clock offsets $\boldsymbol{T}$ and dynamic parameters $\boldsymbol{Y}$. A total of about 
21700 code observations are used. Another is the sum of code and carrier phase observations, which is commonly used for precise orbit determination. Due to incorporation of carrier phase, ambiguity parameters $\boldsymbol{B}$ have to be taken into account. The amount of observations is twice that of code observations, up to about 43400 .

We analyzed the processing time of setting up normal equation system (i.e. Eq. (22), without including the inversion of the matrix) for conventional method and partitioned sub-matrices method. The tests were performed on a Lenovo T430S notebook with a $2.9 \mathrm{GHz}$ processor and RAM available was limited to $4 \mathrm{~GB}$.

Fig. 2 shows the processing time of different types of observations when using conventional and partitioned sub-matrices method. For code observations only, the gain in efficiency with respect to conventional method was a factor of about 2, while it increased to 3.5 if code and carrier phase observation are used together. If the number of GPS observations increased to two-fold, the processing time increased to 2.5 times for conventional method, but it only increased to 1.4 times for partitioned sub-matrices method. So the partitioned sub-matrices method is significantly more efficient in setting up normal equation system.

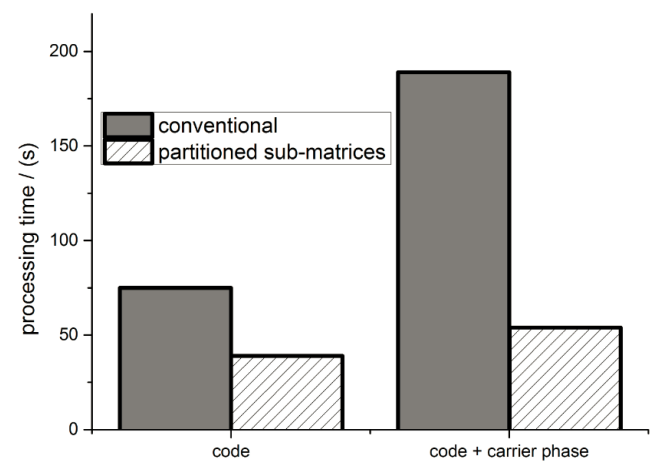

Fig. 2. The processing time comparisons of setting up normal equations for conventional and partitioned sub-matrices modeling method. The left is code observations and the right is sum of code and carrier phase observations.

\section{Enhanced-Accuracy Dynamic Models for Orbit Prediction}

Empirical accelerations, obtained during the orbit determination process, could also be used in orbit prediction. The errors of the atmospheric drag model are the main factor that deteriorates the prediction accuracy for LEO satellites. The drag model depends on the atmospheric density $\rho$ at the satellite location, which strongly varies with the solar flux and geomagnetic activity. The solar and geomagnetic indices would not be predicted precisely during a period of solar maximum. While these indices vary smoothly for short-term predictions during a solar minimum, the errors of the atmospheric drag model show similar characteristics. The empirical accelerations of the present day would likely be used to compensate mismodeling of drag model for next few days. So this paper proposes an "enhanced-accuracy" orbit prediction method which is applied during a period of low solar activity.

The method consists of two steps. First, GPS code observations and ultra-rapid GPS ephemerides are used to determine the satellite states and dynamic parameters of present day. Second, empirical accelerations are modeled by Fourier series and then the fitted empirical accelerations, together with the deterministic force models, form enhanced-accuracy dynamic models that are used for orbit prediction. The orbit prediction scheme is shown in Fig. 3. Four days (July, 3 to 6 2006) GPS code observations, which are chosen during the low solar activity, were selected to test the accuracy of orbit prediction. The GPS rapid orbits and clock corrections of the corresponding days from IGS center ${ }^{16)}$ were used to process GPS data over arc length of 24 hours.

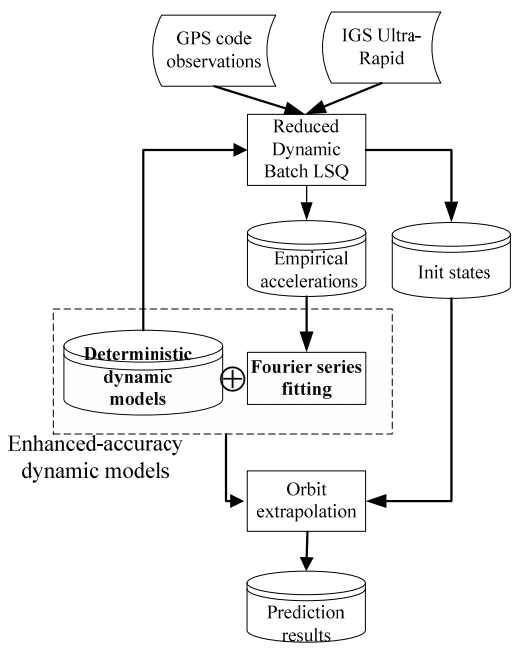

Fig. 3. Processing scheme for orbit prediction.

The satellite position, velocity, $C_{D}, C_{R}$, and empirical accelerations were obtained in step one. Position accuracy was about $0.2 \mathrm{~m}$ and velocity was better than $1.0 \times 10^{-4} \mathrm{~m} / \mathrm{s}$, and the mean value of $C_{D}$ and $C_{R}$ was 3.3 and 1.5, respectively. Figure 4 shows the empirical accelerations in along-track direction that are obtained from GPS code observations on 3 January, 2006. The accelerations are found to have a zero mean and the magnitudes of accelerations are all less than 30 $\mathrm{nm} / \mathrm{s}^{2}$. Other days had similar results.

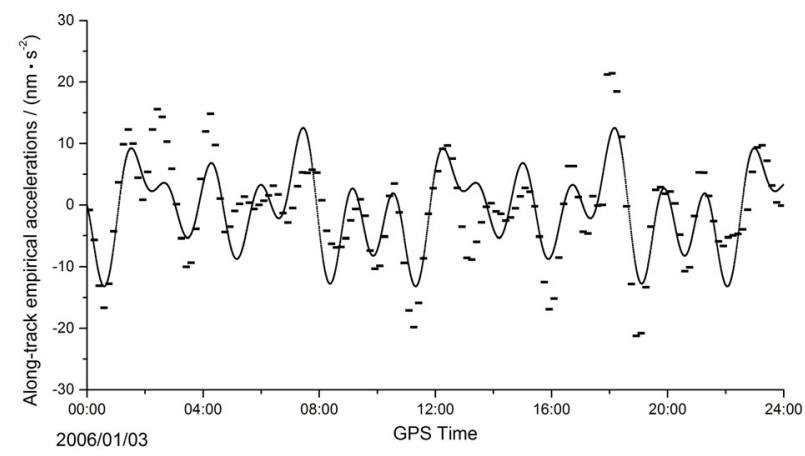

Fig. 4. The estimated empirical accelerations of along-track direction (discrete lines) and the fitted Fourier series curve (Jan. 3, 2006).

Fourier series are applied to fitting along-track empirical accelerations with respect to the time variable. The generalized Fourier series are given by 


$$
f(t)=a_{0}+\sum_{i=1}^{m}\left(a_{i} \cos (i \cdot t \cdot \omega)+b_{i} \sin (i \cdot t \cdot \omega)\right) .
$$

Because unmodeled atmospheric drag is complicated, Fourier series do not always fit well with empirical accelerations in both period and amplitude. The orbital environment of the next few days is not completely the same as that of the present day. The magnitude of unmodeled atmospheric drag might have small perturbation, but its period varies very little over the next few days. Therefore the choice of Fourier series order depends more on how well the period matches that of the empirical accelerations than on its agreement to the amplitude of the empirical accelerations. The period is more important than amplitude for orbit prediction. Take this data for example, the order $m$ is 8 , constant deviation $a_{0}$ equals $-0.693, \omega$ equals 0.5855 , and other coefficients are list in Table 2. Fig. 4 shows Fourier fitting curve, we notice that they are fitting well in period. Similar method could be adapted to other days.

Table 2. The fitted empirical acceleration parameters in along-track direction for Fourier series (Jan. 3, 2006).

\begin{tabular}{|l|l|l|l|l|l|l|l|l|}
\hline$i$ & 1 & 2 & 3 & 4 & 5 & 6 & 7 & 8 \\
\hline$a_{i}$ & -1.71 & -2.44 & 0.53 & -2.33 & 0.30 & 1.27 & 4.31 & 0.72 \\
\hline$b_{i}$ & 1.01 & 2.34 & -0.79 & -3.72 & -0.81 & 1.85 & -3.70 & -0.44 \\
\hline
\end{tabular}

The enhanced-accuracy dynamic models together with the known satellite initial states, starting from the last epoch of that day, are used to extrapolate for next three days. Four group prediction results was validated by reference ephemerides provided by JPL. ${ }^{11)}$

In order to evaluate the effect of this prediction method, a conventional prediction method without considering empirical accelerations models was also tested simultaneously. The accuracy comparisons are shown in Fig. 5. The error of along-track direction increased greatly with respect to prediction time. An extrapolation over 3 days increased the maximum along-track errors up to $150 \mathrm{~m}$ for conventional method. Comparatively, all errors were less than $60 \mathrm{~m}$ for the enhanced-accuracy method. The mean prediction accuracy was improved to 2.3 times when compared with conventional method. The tests showed that the fitted along-track accelerations curve enhanced the precision of the known atmospheric drag models.

\section{Conclusion}

The paper proposes an effective modeling method for empirical accelerations to be used in the reduced dynamic models for determining the orbits of LEO satellites. This method is extremely time-saving and requires less storage for setting up normal equation system when compared with conventional method. This modeling includes an effective computing methods for the sensitivity matrices and partitioning methods for the normal equation. Furthermore, quasi-periodic characteristics of empirical accelerations are fitted with a Fourier series. The fitted empirical acceleration curves can absorb deficiencies of deterministic force models to some extent and improve orbit prediction precision.

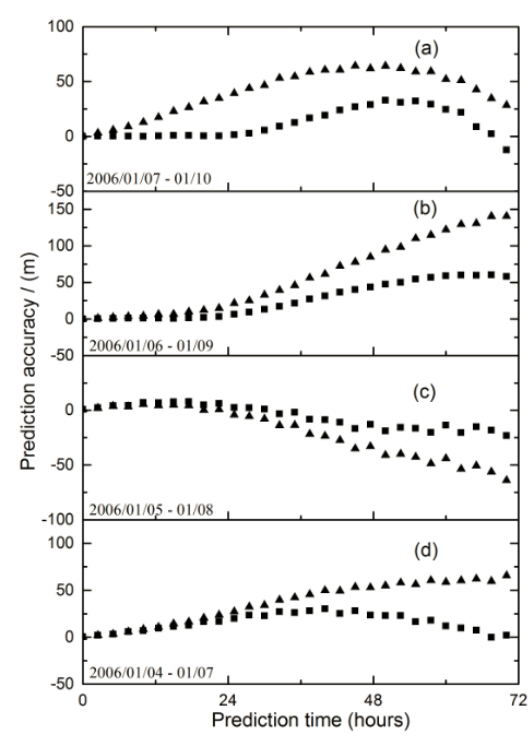

Fig. 5. The comparisons for the along-track deviation. The "triangle" denotes the conventional prediction method without considering empirical accelerations model of along-track direction and the "square" stands for the enhanced-accuracy prediction method. The extrapolation interval is 3 days and start time is Jan. 4(d), 5(c), 6(b), and 7(a), respectively.

Because the majority of the variational equations related to empirical acceleration parameters are homogeneous, sensitivity matrices can be obtained by multiplication of state transition matrices and constant matrices, in other words, they need not numerical integration. The scale of numerical integration and data storage for the method grows linearly with time whereas the conventional method grows quadratically with time. The GRACE-A real flight GPS observation data has been used to evaluate the processing time of setting up normal equation using the proposed and conventional methods. The efficiency increased to 2 times when using code observations only, and it increased to 3.5 times when using both code and carrier phase observations. The more observations used, the higher efficiency obtained.

Empirical accelerations obtained during the orbit determination process have a quasi-periodic nature and accelerations of next a few days often show similarity with those of the present day. So the feature can be successfully applied to orbit prediction if space environment does not vary greatly. The along-track components of the empirical accelerations are interpolated by Fourier series and the periodic features are captured by sine or cosine functions with varying period. Real GPS flight data has been used to verify the orbit prediction accuracy of the enhanced-accuracy dynamic model, which contains both deterministic force models and the fitted empirical acceleration curves. For a 3-day orbit test, the results showed that prediction accuracy was improved to 2.3 times averagely when compared with the conventional method. It demonstrates that the enhanced-accuracy dynamic model is effective and atmospheric drag model errors are absorbed to some extent. It should be noted that the fitted empirical acceleration curves 
are only valid for short-term (such as 3 days) orbit prediction. If the empirical accelerations are applied to long-term orbit prediction without frequently updating the fitted parameters, the predictions will likely be invalid.

\section{Acknowledgments}

We acknowledge the NASA/DLR GRACE project for the GRACE data products and reference ephemerides (distributed by JPL PODAAC). GPS precise and ultra rapid ephemerides are provided by CODE and IGS, respectively. In addition, our deepest gratitude goes to the anonymous reviewer for his carefully and thoughtful suggestions that have helped to improve this paper substantially.

\section{References}

1) Kroes, R.: Precise Relative Positioning of Formation Flying Spacecraft using GPS, Ph.D. Thesis, Delft University of Technology, 2006.

2) Beutler, G., Jäggi, A., Hugentobler, U. and Mervart, L.: Efficient Satellite Orbit Modelling using Pseudo-Stochastic Parameters, J. Geod., 80 (2006), pp. 353-372.

3) Wu, S. C., Yunck, T. P. and Thornton, C. L.: Reduced-dynamic Technique for Precise Orbit Determination of Low Earth Satellites, $J$. Guid. Control Dyn., 14 (1991), pp. 24-30

4) Visser, P. and Van den IJssel, J.: Aiming at a 1-cm Orbit for Low Earth Orbiters: Reduced-dynamic and Kinematic Precise Orbit Determination, Space Sci. Rev., 108 (2003), pp. 27-36.
5) Swatschina, P.: Dynamic and Reduced-dynamic Precise Orbit Determination of Satellites in Low Earth Orbits, Ph.D. Thesis, Vienna University of Technology, 2009.

6) Montenbruck, O., Van Helleputte, T., Kroes, R. and Gill, E.: Reduced Dynamic Orbit Determination using GPS Code and Carrier Measurements, Aerosp. Sci. Tech., 9 (2005), pp. 261-271.

7) Wang W. B. and Liu R. F.: Precise Orbit Determination Based on Reduced Dynamic Batch LSQ Estimation Method Using Dual-frequency GPS Observations, Chin. J. Space Sci., 34 (2014), pp 460-467 (in Chinese).

8) Jäggi, A., Bock, H. and Floberghagen, R.: GOCE Orbit Predictions for SLR Tracking, GPS Solut., 15 (2011), pp. 129-137.

9) Colombo, OL.: The dynamics of Global Positioning Orbits and the Determination of Precise Ephemerides, J. Geophys. Res., 94 (1989), pp. 9167-9182.

10) GRACE Level 1B Products, ftp://podaac.jpl.nasa.gov/allData/grace/, (cited on 21 November 2012).

11) High Rate Clocks and Orbits, ftp://ftp.unibe.ch/aiub/CODE (cited on 8 January 2014).

12) GRACE Gravity Model, ftp://ftp.csr.utexas.edu/pub/grav, (cited on 3 December 2013).

13) Montenbruck, O. and Gill, E.: Satellite orbits: models, methods and applications, Springer Verlag, Berlin, 2000.

14) Picone, J. M., Hedin, A. E., Drob, D. P. and Aikin A. C. NRL-MSISE-00 Empirical Model of the Atmosphere: Statistical Comparisons and Scientific Issues, J. Geophys. Res., 107 (2002), pp.SIA15-1 - SIA15-16.

15) Knocke, P. C., Ries, J. C. and Tapley, B. D.: Earth Radiation Pressure Effects on Satellites, Proceedings of the AIAA/AAS Astrodynamics Conference. Minneapolis, Minnesota, USA, 1988.

16) Dow, J., Neilan, R. E. et al.: The International GNSS Service in A Changing Landscape of Global Navigation Satellite Systems, J. Geod., 83 (2009), pp. 191-198. 